\title{
Analyzing Predator-Prey Model
}

\author{
Laxman Bahadur Kunwar \\ Thakur Ram Multiple Campus, Tribhuvan University \\ Email: laxmankunwar23@gmail.com
}

\begin{abstract}
In this article, we consider a system involving two-species living in the same environment and describe the model for their population growth presented by Lotka and Volterra.The model is the foundation for the development of many other models. The model is known as PredatorPrey Model or Lotka-Volterra system. In more modern theories, there will be multiple species each with their own interactions but we will limit ourselves this to simpler but highly instructive classical system. We shall cover various standard tools for analysing such systems. We shall discuss dynamic solutions, equilibrium solutions and phase curves that best illustrate the phenomena.
\end{abstract}

Keywords : predator, prey, equilibrium condition, lotka-volterra model.

\section{Introduction}

Thomas R. Malthus(1798), a British Economist, purposed a mathematical model of the population growth for single species, called Malthusian law of population growth which is widely regarded as the first principle of population dynamics in the field of population ecology. The law is also called the exponential population model. The law is subsequently modified by Verhulst(1833). Lotka(1925) and Volterra(1931), considered two populations i.e. two species and analysed the model. They formulated the model after considering predatorprey of situations. Many researchers have studied the techniques as predator-prey interactions, mutualisms and competitive mechanisms and made an attempt to develop a more biologically realistic model.

Predator-Prey models are arguably the building blocks of the bio- and ecosystems as biomasses are grown out of their resource masses. Species compete, evolve and disperse simply for the purpose of seeking resources to sustain their struggle for their existence. Depending on their specific settings of applications, they can take the forms of resource-consumer, plantherbivore, parasite-host tumour cells (virus)-immune system, susceptible-infectious interactions, etc. They deal with the general loss-win interactions and hence may have applications outside of ecosystems. When seemingly competitive interactions are carefully examined, they often in fact some forms of predator-prey interactions in disguise. 
Lotka-Volterra model is a pair of differential equations representing the populations of a predator and prey species which interact with each other in same environment. So predatorprey model of a simple eco-system is an application of the non-linear system of differential equations in mathematical biology/ecology. The model was independently proposed by American statistician Alfred J. Lotka and Italian mathematician Vito Volterra.( Anisiu,2014)

\section{Predator-Prey Model}

Lotka-Volterra model is composed of a pair of differential equations that describe predator prey dynamics in their simplest case .(one predator population and one prey population). The model is characterized by oscillation of the population size of both predator and prey, with the peak of the predator's oscillation lagging slightly behind to peak of the prey's oscillation. The model makes several simplifying assumptions (SagayaRaj et al.,2013) :

(i) Prey population grows exponentially when the predator is absent.

(ii) Prey death rate is proportional to the size of both the predator and prey population.

(iii) Predator population starves to death in the absence of the prey.

(iv) Predator can consume infinite qualities of prey.

(v) There is no environmental complexity.

Predator and prey can influence one another's evolution. Population ecology is dominated by the study on interspecies interactions such as competition and predation. Population change of a species depends on its reproduction rate and the interaction with other species. Population size of prey, say greenflies, is a time-dependent function and denoted by $x$. If the food supply of this species is unlimited, it seems reasonable that the rate of growth of this population would be proportional to the current population size, as there are more potential couplings (Malthus, 1798) so

$\frac{d x}{d t}=\beta_{1} x \Rightarrow x(t)=A e^{\beta_{1} t}$

Where $\beta_{1}=$ the growth rate of prey population

$\mathrm{A}=x(0)$, the initial population size of prey.

$$
\frac{d x}{d t}=\text { growth rate of prey population with respect to time. }
$$

The clear problem with this model is that the population grows without bound over time which is not true in the real world. One method to correct this problem is that one might specify that the growth rate $\beta_{1}$ becomes a function of the population size, decreasing as $x$ increases. Alternatively, we could model a second population $y(t)$ which represents a second species, say ladybirds, which prey on the greenflies. In this case, the greenfly population $x$ will decrease proportionally to the number of ladybirds y multiplied by the number of greenflies $x$, 
i.e. the number of interactions of the two species which may lead to a sad little greenfly funeral. This law will be in the form

$$
\frac{d x}{d t}=\beta_{1} x-c_{1} x y
$$

Where, $c_{1}$ is the rate at which fatal interactions occur. But in such case, the changing predator population $y(t)$ must also be modelled. We assume that in the absence of prey, it will decrease as its food supply will be shortage. So

$$
\frac{d y}{d t}=-\alpha_{2} y
$$

Where, $\alpha_{2}$ - Predator mortality rate.

$\frac{d y}{d t}$ - Growth rate of predator population with respect to time.

But in the presence of prey, the decrease in the population of predator is opposed by the predator birth rate. The predator birth rate is determined by the product of the number of predator $(y)$ times the number of prey $x$ and by the predator's ability to turn food into offspring. So

$$
\frac{d y}{d t}=-\alpha_{2} y+c_{2} x y
$$

Where, $c_{2}-$ Growth rate of predator or predator ability at turning food into offspring. As a consequence of these assumptions, the population change through time is represented by the set of two ordinary differential equations

$$
\begin{aligned}
& \frac{d x}{d t}=\beta_{1} x-c_{1} x y \\
& \frac{d y}{d t}=-\alpha_{2} y+c_{2} x y
\end{aligned}
$$

This system of equations is called Lotka-Volterra (predator-prey) system. In more modern theories there will be multiple species each with their own interactions but we will limit ourselves to this simpler but highly instructive classical system.

\section{Discussion}

The model predicts a cyclical relationship between predator and prey numbers as the number of predator $(y)$ increases so does the consumption rate $\left(c_{2}\right)$, tending to reinforce the increase in $y$.Increase in consumption rate, however, has an obvious consequence a decrease in the number of prey $(x)$, which in turn causes $y$ to decrease. Now $y$ can increase, and the cycle begins again. The graph 3.1 shows the cyclical relations predicted by the model for hypothetical predator and prey populations. 


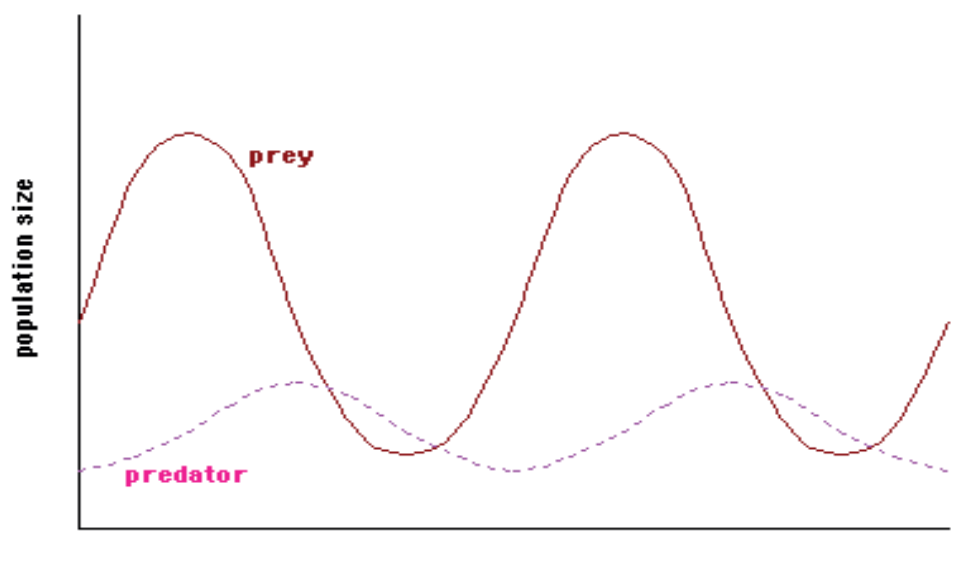

time (t)

Fig 3.1, Hypothetical Predator and Prey populations.

3.1 The Equilibrium Points: At the equilibrium point, $\frac{d x}{d t}=0, \frac{d y}{d t}=0$

$x \beta_{1}-c_{1} x y=0 \quad \Rightarrow \quad x\left(\beta_{1}-c_{1} y\right)=0$

and $-\alpha_{2} y+c_{2} x y=0 \quad \Rightarrow \quad y\left(-\alpha_{2}+c_{2} x\right)=0$

These two equations being nonlinear differential equations, they may represent closed circles or other shapes like spiral. We shall find all possible solutions.

Case (i) By equation (i) either $x=0$ or $\beta_{1}-c_{1} y=0$

If $x=0$, then putting in equation (ii), we get $y\left(-\alpha_{2}+c_{2} x\right)=0$

$\Rightarrow y=0$

If $\beta_{1}-c_{1} y=0$ then $y=\frac{\beta_{1}}{c_{1}}$

By equation (ii) we get $\frac{\beta_{1}}{c_{1}}\left(-\alpha_{2}+c_{2} x\right)=0$

$\Rightarrow x=\frac{\alpha_{2}}{c_{2}}$

Hence the solutions are $(0,0)$ and $\left(\frac{\alpha_{2}}{c_{2}}, \frac{\beta_{1}}{c_{1}}\right)$.

Case (ii) Similarly, taking equation (ii ) first and then putting in equation ( $\mathrm{i}$ ), we get the same solutions $(0,0)$ and $\left(\frac{\alpha_{2}}{c_{2}}, \frac{\beta_{1}}{c_{1}}\right)$. 
So $(0,0)$ and $\left(\frac{\alpha_{2}}{c_{2}}, \frac{\beta_{1}}{c_{1}}\right)$ are the two equilibrium points.

We have $x=0, y=0, y=\frac{\beta_{1}}{c_{1}}$ and $x=\frac{\alpha_{2}}{c_{2}} \quad$ These are nullclines. These lines divide the phase plane into the four regions in which the trajectories have different directions.

\section{Direction of Trajectories}

The Lotka-Volterra equations can be written as $\frac{\mathrm{dx}}{\mathrm{dt}}=\mathrm{c}_{1} x\left(\frac{\beta_{1}}{\mathrm{c}_{1}}-\mathrm{y}\right)$

$$
\frac{d y}{d t}=c_{2} y\left(-\frac{\alpha_{2}}{c_{2}}+x\right)
$$

\begin{tabular}{|l|c|c|l|}
\hline Region & Values of $\mathbf{x}$ and $\mathbf{y}$ & Sign of derivatives & Direction of $\mathbf{x}$ and y \\
\hline I & $x>\frac{\alpha_{2}}{c_{2}}>0$ & $\frac{d x}{d t}<0$ & $\mathrm{x} \downarrow$ \\
& $y>\frac{\beta_{1}}{c_{1}}>0$ & $\frac{d y}{d t}>0$ & $\mathrm{y} \uparrow$ \\
\hline II & $0<x<\frac{\alpha_{2}}{c_{2}}$ & $\frac{d x}{d t}<0$ & $x \downarrow$ \\
& $y>\frac{\beta_{1}}{c_{1}}>0$ & $\frac{d y}{d t}<0$ & $y \downarrow$ \\
\hline III & $0<x<\frac{\alpha_{2}}{c_{2}}$ & $\frac{d x}{d t}>0$ & $x \uparrow$ \\
& $0<y<\frac{\beta_{1}}{c_{1}}$ & $\frac{d y}{d t}<0$ & $y \downarrow$ \\
\hline IV & $x>\frac{\alpha_{2}}{c_{2}}$ & $\frac{d x}{d t}>0$ & $x \uparrow$ \\
& $0<y<\frac{d y}{c_{1}}$ & $\frac{d y}{d t}>0$ & \\
\hline
\end{tabular}




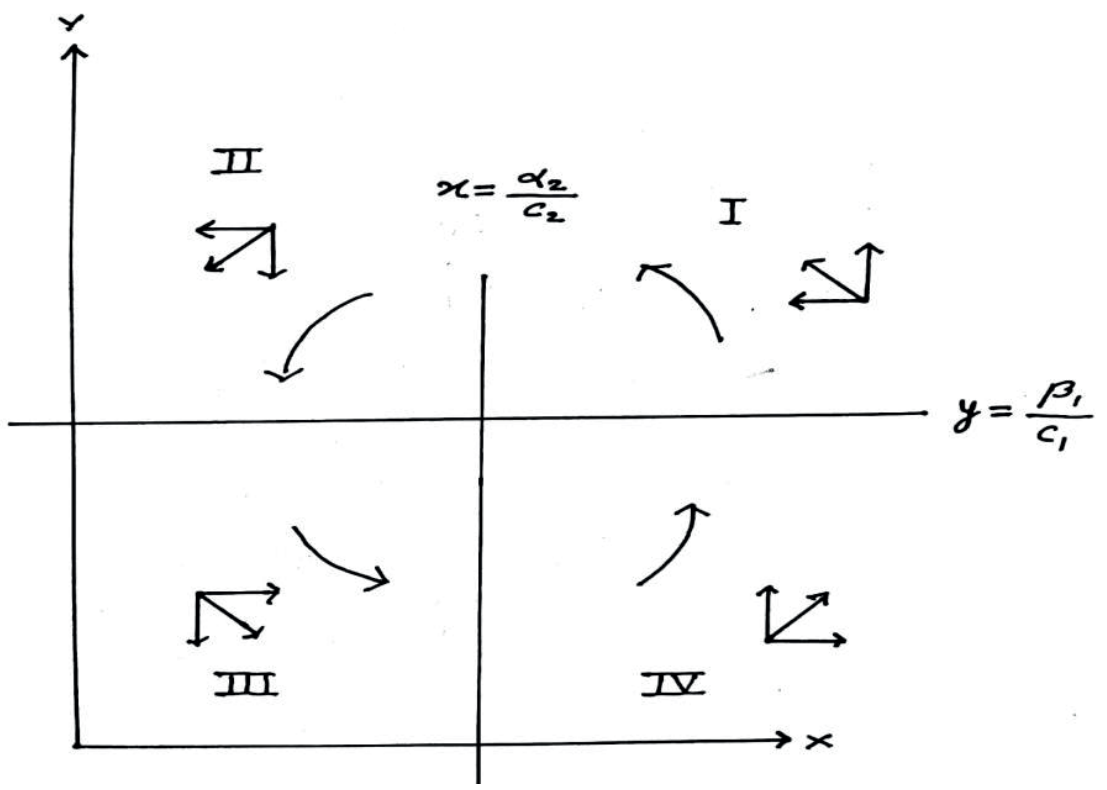

Fig 3.2 The Resultant directions of the trajectory.

The directions of $x$ and $y$ in the four regions tell us that we have to trace out trajectory in Anticlockwise direction. But it does not tell us that whether this is spiral or a closed trajectory.

\subsection{Finding the Trajectory:}

Eliminating t by using chain rule

$\frac{d y}{d x}=\frac{-\alpha_{2} y+c_{2} x y}{\beta_{1} x-c_{1} x y}$

$\Rightarrow \frac{d y}{d x}=\frac{y\left(-\alpha_{2}+c_{2} x\right)}{x\left(\beta_{1}-c_{1} y\right)}$

$\Rightarrow \frac{\left(\beta_{1}-c_{1} y\right)}{y} d y=\frac{\left(-\alpha_{2}+c_{2} x\right)}{x} d x$

$\Rightarrow\left(\frac{\beta_{1}}{y}-c_{1}\right) d y=\left(\frac{-\alpha_{2}}{x}+c_{2}\right) d x$

$\Rightarrow \beta_{1} \ln (y)-c_{1} y=-\alpha_{2} \ln (x)+c_{2} x+K_{1}$

Where, $K_{1}$ is the constant of integration. This is the equation of trajectory. Now we shall show that it represents a closed curve. 
Taking a straight line $x=x_{1}$ (constant)

Dividing each term of the equation ( iii) by $\beta_{1}$

$$
\begin{aligned}
\ln (y) & =\frac{c_{2}}{\beta_{1}} y-\frac{\alpha_{2}}{\beta_{1}} \ln \left(x_{1}\right)+\frac{c_{2}}{\beta_{1}} x_{1}+\frac{K_{1}}{\beta_{1}} \\
\Rightarrow \ln (y) & =\frac{c_{2}}{\beta_{1}} y+K
\end{aligned}
$$

Where $K=-\frac{\alpha_{2}}{\beta_{1}} \ln \left(x_{1}\right)+\frac{c_{2}}{\beta_{1}} x_{1}+\frac{K_{1}}{\beta_{1}}$, a constant term.

Putting $\quad z=\ln (y) \quad$ we get $\quad \mathrm{z}=\frac{c_{2}}{\beta_{1}} y+K$.The graph of $\mathrm{z}$ against $Y$ for these parametric equations are logarithmic curve and a straight line intersecting at two points only. If it had a spiral curve there will be infinite number of intersection points with line $x=x_{1}$. But if the curve is a closed curve it will intersect at most two points. Hence the trajectory is a closed curve.

It is observed that suddenly disturb will form new trajectory. All Phase plane curves are traced in anticlockwise direction along closed curve enclosing equilibrium point. Prey population $(x)$ reaches the maximum before predator population $(y)$. Thus the predator oscillation cycle always lags behind the prey oscillation.

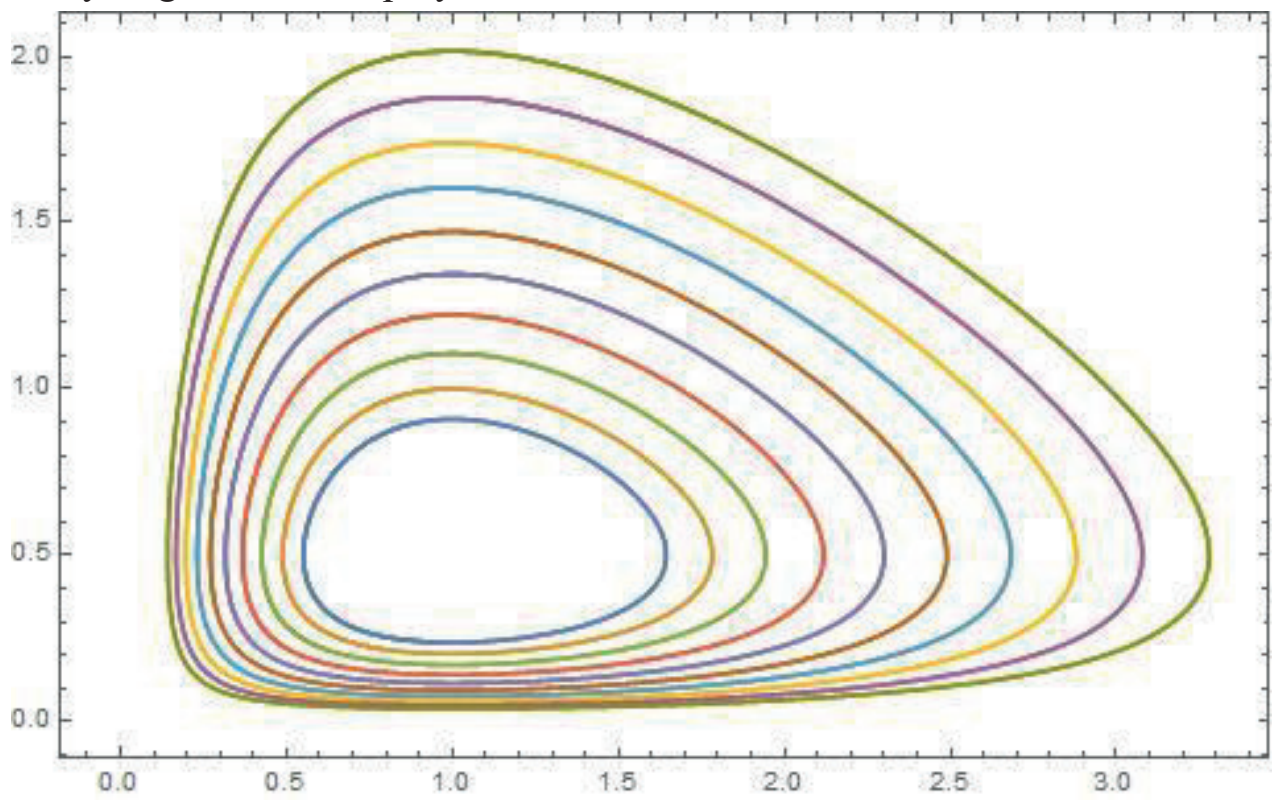

Fig.3.3 Trajectories of predator-prey model for different values of constant. 


\section{Limitations of the Model}

a) The model is limited to laboratory typed environment as we have neglected many other factors such as harvesting, time delay, diseases, affects of third species etc.

b) The model doesn't consider competition among prey or predators themselves.

c) It assumes that prey population may grow infinitely without any resources limits.

d) It does not consider the concept of the carrying capacity.

e) Predators have no saturation. Their consumption rate is unlimited.

\section{Conclusions}

Lotka-Volterra model is the first and simplest predator-prey model involving two species; one of them the predator, feeds on the other species (the prey). The prey in turn feeds on some third food available around the environment. It is observed in the phase plot that there is a periodic relationship between the two parameters. The population at which equilibrium occurs will depend on the value of the constants which are biologically determined parameters. The problems with the predator-prey equations are discussed and their causes are also explained. But the model is limited to laboratory typed environment as we have neglected many other factors such as harvesting, time delay, diseases, affects of third species etc. The model can be extended by including three or more species and factors influencing the population of predator as well as prey.

The model is a mathematical formulation of the predator prey form of interactions and can be used to predict population behaviour of various species at different times. After analysing some population data of species of forest, one can estimate an average time of periodic oscillations. This information can be incredibly useful to the biological fields focused on extinction prevention and co evolution studies. After determining the population fluctuation characteristics, the results can show extinction prevention specialists the year and seasons where the extinction is naturally possible, preparing them ahead of time to do intense tagging and developing natural habitats as safety precautions. Co evolution studies could also apply this model to determine the number of cycles it required for each species to develop new means of survival and adapt to the competition. Also infinite number of diverse and complex models could be developed using this classical model as a basic starting points. 


\section{References}

Anisiu, M.C. (2014). Lotka, Volterra and Their Model. Didactica mathematica, Vol.32, pp.917

Devaki,K.B. \& Swathis, S. (2016). The system of Differential equations in Prey Predator Model. International journal of multidisciplinary Research and Modern Education, Vol.2(1) .

Malthus,T.R.(1798). An Essay on te Principal of Population, J. Johson in St. Paul's Churchyard, London.

Murray,J.D. (2004). Mathematical Biology I: An Introduction, 3-e, springer international edition.

Sagaya Raj, M.R. , Maria, A. G. and Janagaraj, R.(2013). Stability in a Discrete Prey-Predator Model. International Journal of Latest Research in Science and Technology, Vol.2(I). pp. 482-485.

Verhulst,P.F.(1833). Notice sur la loi que la population suit dans son accrissement, in : J.G. Garnier, A. Quetelet (Eds) Correspondance Mathematique et Physique, Vol.10, Observatoure royal de sedgique ,pp.113-121.

Volterra,V.(1931). U.D'Ancona, La concorrenza vitaletra le specie dell 'ambiente marino, in(1931) VIIe Congre,s International d' Aquaculture et de Peche, Paris1931, Societe Nouvelle de I'Imprimerie du Loiret, Orleeans, pp.1-14. 